\title{
A discussion about the potentials and pitfalls of quantitative nuclear magnetic resonance (qNMR) spectroscopy in food science and beyond
}

Dirk W. Lachenmeier ${ }^{a \star}$, Torsten Schönberger ${ }^{b}$, Sebastian Ehni ${ }^{c}$, Birk Schütz ${ }^{d}$, Manfred Spraul ${ }^{d}$

${ }^{a}$ Chemisches und Veterinäruntersuchungsamt Karlsruhe, Weissenburger Strasse 3, 76187 Karlsruhe, Germany.

Corresponding Author: Lachenmeier@web.de

${ }^{\mathrm{b}}$ Bundeskriminalamt, Kriminaltechnisches Institut, KT 12, 65173 Wiesbaden, Germany.

E-Mail: torsten.schoenberger@bka.bund.de

${ }^{c}$ ChemCon GmbH Freiburg, Engesserstr. 4b, 79108 Freiburg im Breisgau, Germany. E-Mail: Sebastian.Ehni@chemcon.com

${ }^{d}$ Bruker Biospin GmbH, Siberstreifen, 76287 Rheinstetten, Germany. E-Mail: Manfred.Spraul@bruker.com;

Birk.Schuetz@bruker.com

During the XIII International Conference on the Applications of Magnetic Resonance in Food Science, which was held in Karlsruhe, Germany, from 7th to 10th of June 2016, a discussion session entitled "Quantitative nuclear magnetic resonance (qNMR)" was organized. The conference participants had the opportunity to submit written questions as well as to ask ad-hoc questions during the session, which were to be answered by a panel of qNMR experts from several application fields. This article provides an edited and referenced transcript of the session. The major topics were centred on instrumental requirements for qNMR, and the participants were in agreement that modern digital NMR spectrometers guarantee a long-term stability of measurements and calibrations, sometimes over several years. However, the panel also agreed that method validation is an absolute necessity in qNMR as in every other field of quantitative analytical chemistry. Validation strategies may depend on the purpose of the method and vary between multi-component analyses of foods and beverages, compared to single target assays e.g. in verification of reference standards. Approaches to establish limits of detection and to ensure the required method accuracy were suggested. The discussion was closed with a general agreement of the experts that qNMR in food science will gain a wider application range in the future. The necessity to abolish regulatory hindrances - including the approval of using qNMR in reference methods - was stressed.

\section{Introduction}

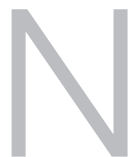

uclear magnetic resonance (NMR) spectroscopy has occasionally been used for quantification since the 1960s (see Figure 1 and the overview in Bharti \& Roy ${ }^{1}$, however only the advancements of digital spectrometer technology during the last two decades have made this technique available for routine analysis in competition to the commonly used chromatographic methods for quantification of compounds in food science. Only very few applications of quantitative NMR (qNMR) were published till the mid-1990s, and since then an exponential growth in development of applications in this area can be seen (Figure 1), which still has not reached a saturation or plateau. Reviews about the basic principles of qNMR are available from Bharti \& Roy ${ }^{1}$ and Simmler et al ${ }^{2}$.

The discussion session entitled "Quantitative nuclear magnetic resonance (qNMR)" at the XIII International Conference on the Applications of Magnetic Resonance in Food Science (MR in Food 2016, Karlsruhe, Germany, 7th to 10th of June 2016, Figure 2) was chaired by Dirk W. Lachenmeier (DL), a food chemist and toxicologist, having worked with qNMR in governmental food control since $2003^{3,4}$

The participating experts were:

- Torsten Schönberger (TS) who works for the Bundeskriminalamt (BKA) in Wiesbaden, Germany, at the Forensic Science Institute. His main interests with qNMR are not only the analysis of drugs including illicit drugs but also pharmaceuticals, doping, plastics, and explosives, among other forensic materials ${ }^{5-8}$.

- Sebastian Ehni (SE) who works at ChemCon GmbH in Freiburg, Germany, a manufacturer of pharmaceutical active ingredients to be applied at humans. SE is using NMR spectroscopy and quantitative NMR spectroscopy in quality control ${ }^{9-11}$.

- Manfred Spraul (MS), the chief technology officer of the new Applied Industrial Clinical (AIC) division of Bruker BioSpin, Rheinstetten, Germany. AIC includes not only a large part of food science developments but also clinical applications and other areas such as cosmetics that might be included in the future ${ }^{12-16}$.

- Birk Schütz (BS), the head of the method development team for food analysis, also from Bruker BioSpin ${ }^{17-19}$.

The following text provides a transcript of the discussion, which was edited and referenced by the experts.

\section{History and instrumental aspects of qNMR}

DL: I want to start the discussion by asking Dr. Spraul to give us a short overview of the history of quantitative NMR. Is quantitative NMR only a recent development?

MS: Well of course, quantitative NMR was done for a long time with the main focus, of course, to detect impurities, e.g. of drugs or other chemicals. What is relatively new, relative you would say might be 10 years, is really the development of looking into mixtures. And this is, I would say, just as infrared spectroscopy has also 


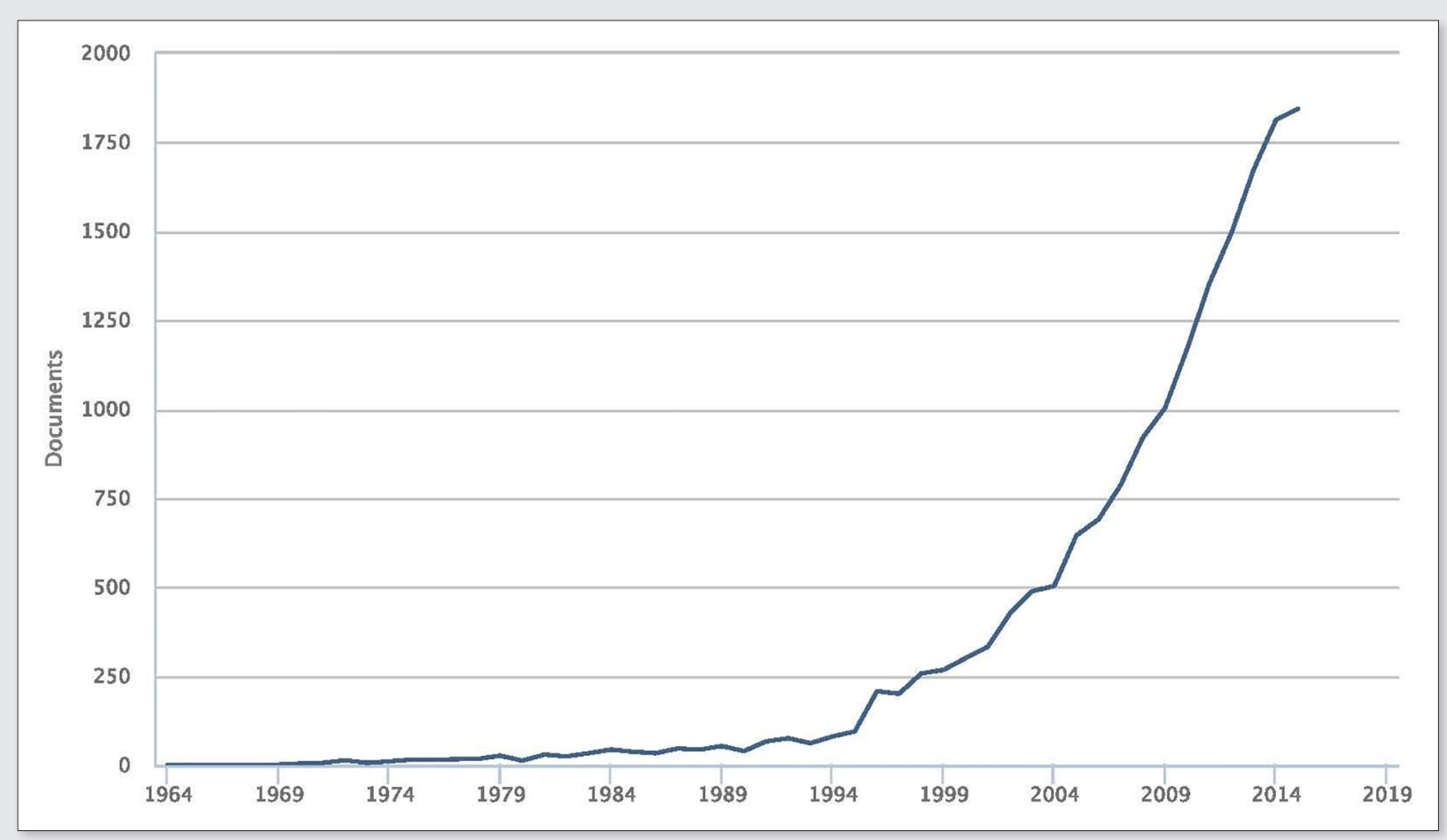

Figure 1. Number of scientific publications 1991-2015 on applications of qNMR in food science. Source: Scopus, Elsevier B.V.; search term: "(quantitative nuclear magnetic resonance OR qNMR) AND (food*)", search conducted June 17, 2016.

been taking, that also started with structure identification and has strongly moved into mixture analysis and this is where NMR is moving in as well. And of course, for foods this is basically what we need to do here to look into mixtures. Now, why is this possible? The first requisite I think is the instrument, and we have to make sure that the spectral quality that is coming out of that is good enough.

So that means, for example, a high dynamic range of the receiving system. So the introduction of the digital spectrometers was a major step towards a higher quality. So this is the first prerequisite, the receiving system, but also we have to look into flat baselines, because if we look into a mixture, it's more difficult to quantify if you have to subtract a rolling background or whatever might have been there in the years before. So a lot of effort has been taken, for

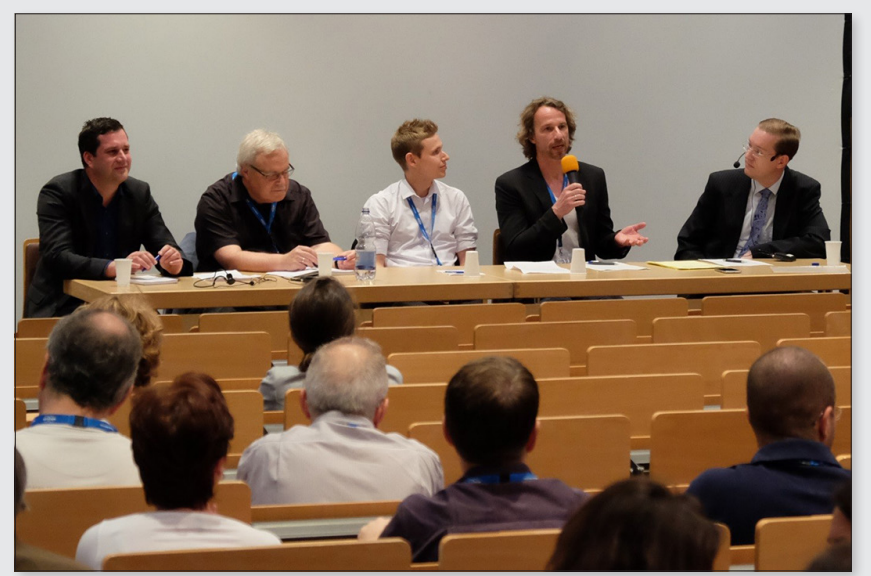

Figure 2. Photograph of the experts participating in the gNMR discussion (from left to right: Birk Schütz, Manfred Spraul, Sebastian Ehni, Torsten Schönberger, and Dirk W. Lachenmeier, chair) example, in digital filtering to make sure that the spectra, which you can achieve now based on certain conditions of the parameters that you choose, really deliver you an absolutely flat baseline. The next point, that had to be solved, is in the food matrix.

Water suppression is the main thing that we have to achieve. This was also an issue because if the residual phase of the water signal that you suppress is different than the phase of the signals you want to quantify, then you have your signals eventually on a steep curve, and then it gets difficult again. So this is another important development these days with the standard operation procedures (SOPs) that we also provide. We can really make sure that flat baseline, and in addition also this residual phase is absolutely consistent. And when we look in our food screeners, for example, where we offer remote service and analyses for other companies remotely, we don't look at the spectra anymore. We are sure that the quality is what it was supposed to be.

When we started, for example, with Jeremy Nicholson in the body fluid arena many years ago ${ }^{20}$, and even until let's say the year 2000 or a bit later, we had to basically re-process manually about $30 \%$ of the samples, and of course this is not acceptable for highthroughput screening as we need it in the food area. This is why we spend a lot of time sorting that problem. Then, automation is extremely important, because what we also can see might be less in the quantification but it will be there for sure, if we have taken, for example, statistical data and we have looked to who has baseline corrected and done all this, you could actually do a statistic which differentiates the person that has actually processed the data.

And this is not good, because we have to keep the instrumental variability very small to be sure that we can actually track small changes in concentration. This is even more important in multivariate statistics. That's why at Bruker, we would not consider NMR as a method that only does quantification, because there are other 
methods which have much lower prices, and you have to justify added value to actually sell such an expensive system, first of all, into this market. In the food market people are not used to prices... Like for example, up to 600,000 Euro is difficult to justify. So that's why we have to choose an instrument which is moderate in price and we need an automation which is reliable. Next thing which is absolutely vital for success is the SOPs. Of course, now we can say if you looked to individual chemicals, you can't do that, that easily, but in the food arena, when we always look, for example, to honey or wine or fruit juice or edible oils, then we have to do this consistently. If we don't do that you will get different quantification results and many other problems are coming up. So I think these were probably the most important developments, just in a short summary.

DL: I think it's quite exciting that analysing over 50 compounds quantitatively from one single spectrum is possible. Perhaps Dr. Schütz wants to expand about this possibility so that not only one compound can be quantified but you can do a lot of compounds and much more than what has been possible with other spectroscopic techniques.

BS: We have developed, starting in 2008 , our fruit juice profiling module, which is able to quantify 40 compounds and to distinguish different types of fruit juice in full automation. In 2010, we started with wine. From there, we have fully automation for 55 compounds. And last year we released the honey profiling, which was only able to quantify 36 compounds, but this is only the quantification part where the major part of those developments are really the spectral data bases behind. For fruit juice, we have currently 29,000 reference samples. In wine, we have selected and measured 19,000, and in honey we have currently something about 8,000 reference spectra. And with those reference databases we are able to identify geographical origin, botanical variety, vintage and things like that, and of course the nontargeted verification we get any deviations which are not typical for this type of sample.

MS: What is also important, if we do this industrially and this is used, it might be also in court. Then, of course, you have a high responsibility that the results that you're generating are correct. That means that you have to know exactly what your matrix is and what the variability in your matrix is, not only for the statistics where it's absolutely vital but also for quantification. We must know what is the typical lowest and highest concentrations that we can tune the system for. And therefore, to run something like 50 samples might be irresponsible. This is not a criticism, please, don't understand that correctly. For scientific publication, I can do that. I can draw my conclusions, but if we are taking responsibility, like in fruit juice, if there is a fruit juice tanker from Brazil coming to Rotterdam and we say based on our measurement that this is not good, you can imagine the value which is behind that. So, there is a huge responsibility that we have to take, and therefore of course this is why we have these 28,000 fruit juice samples and so on. We need this to be absolutely safe.

DL: Dr. Ehni please introduce what is the interest of the pharma industry in quantitative NMR perhaps different to the food industry. SE: In pharma industry we have a goal, usually produce something, and at the end of the day we want the job to be done. The goal would be, for example, to have a product, and the product is good, if it has a certain assay. Let's say 99\%. To establish the assay, HPLC, GC or qNMR are frequently used. At the end of the day it's often the case that a new qNMR method is ready to use while HPLC and GC development takes several more weeks. But one of the key points is that authorities are so far not very used to NMR spectroscopy as they are to HPLC or GC.

DL: Torsten Schönberger, in your field at a criminal investigation lab, perhaps in light of what we know from television, it is quite exciting that NMR can give very fast results. Can you perhaps explain a bit what you are doing with quantitative NMR?

TS: Maybe it's a little bit surprising but our NMR instrument, we have only one, and we are doing much more analyses in our institute than all of the mass spectrometers together, three times more I would say. Our customers, our internal other units, are really used to use the NMR. And in most of the cases we also want to have the quantitative value because we can do this. And of course, we have our own LC/GC applications for quantification of amphetamine for example, but all the other stuff, they cannot analyse because they don't have the procedure for that, they don't have a standard, and so that's our turn now, with the NMR. And we have a lot of, let's say street samples to analyse. And of course, the customer and also the court in the end is interested in getting the content of the active pharmaceutical ingredient (API), because the amount from the seizure of the API is relevant for the punishment in the end. It's our legal system and I think it's for the most European countries the same. But we do that also for intelligence, for profiling, when we want to compare different samples. And we have one procedure where we give purity of analytical standards which other police institutes, forensic science institutes want to use for their chromatographic analysis.

So they cannot go to Sigma-Aldrich or so, and buy the really new psychoactive substance, which is quite new on the market. So we take it from seizure, maybe they do a preparation and a purification step, and then we have to find the purity of that. And we can do this if everything works well with an uncertainty level of $0.15 \%$, something like that. But this is only relevant for the purity determination of these standards.

DL: I think this is one of the very important applications in this field and we, as a food, cosmetics and medicinal products control authority, also have the same problem that for many substances, which we want to quantify, the reference standards are not available or so expensive that we do not want to pay the price. In these cases, NMR is the only method for quantification without having the actual reference standard.

But now I want to start with the questions submitted by the participants of the meeting. There was one question, how the accuracy of external calibrations overtime can be provided and optimized, and especially the question was, how often do I have to recalibrate instrument?

BS: This really depends on the infrastructure. We in our facility, we have one instrument with a very robust infrastructure. There we use one calibration for 1.5 years, but it really depends. It could also be that you have to recalibrate once a month or so. So I think validation, as a daily constant quality control of your NMR system is really necessary, and so we are using an external reference sample, which is composed of five compounds, and through the validation we quantify all the five compounds, check the internal variability if the sample has degraded, and out of this analysis we calibrate the system.

DL: I agree this is the best, or the optimal method. We are also using such a calibration sample, for example, for analysing alcoholfree beverages ${ }^{21,22}$, and we measure this with every sample series 
to be absolutely sure, in addition to the normal validation you have to do anyway to establish such a method.

The second question, perhaps a bit more exotic, was about using DOSY for qNMR of liquid mixtures. Is this something possible, or, is anyone doing this?

MS: Everything is possible. Whether it's useful to do, is another question. So DOSY, you have to use it broadly as a 1D filtered experiment, if you want to do screening, and therefore it might not be the best because you lose signal-to-noise $(\mathrm{S} / \mathrm{N})$, and therefore I think you have to acquire longer, even if you might be more specific on differentiating certain compounds, but overall I think it takes you 2-3 times longer at least to get a reasonable $\mathrm{S} / \mathrm{N}$ that you can work on. The other thing is, the question of diffusion coefficients. How are they changing when concentrations of other compounds are changing? That is, I think not perfectly investigated. It's just another area where you can introduce errors, and our opinion is to make it as simple as possible, but of course test the simplicity very hard, and then you can go and you have less variables which might harm you. That's my answer to this.

DL: There was another interesting question: is it possible to enhance a signal intensity of one component in a multi component mixture? It's not quite clear but I think the question goes in this way that you want to only quantify this one single signal and perhaps suppress the other signals like, we are doing with the alcohol suppression of several signals to improve the detectability of the other minor compounds $^{23,24}$.

MS: What you could do is a selective excitation, for example. That also needs a lot of calibration and might also need a transfer in between, because the signal that you want to see is sitting in a heavily overlapped region, then it makes no sense to excite this region, but it would make sense if the compound has another signal, which is relatively free, to try to do it like this through a selective COSY, TOCSY with short mixing time, and then you have to calibrate the quantification. That's the way I would see it, but again, this is one molecule, in mixtures we normally have to look at many molecules. So there it might not be that useful. If I really go only for one compound, then this is something you have to think about.

DL: I absolutely agree. You also want to detect the outlying samples, where something has happened or has been adulterated or contaminated, and if you only look at one signal this could be perhaps dangerous.

So perhaps a question for you, Dr. Ehni is the next question about the best compromise of using 400,500 or $600 \mathrm{MHz}$ for quantification. What would you suggest as necessary or as useful or as perhaps the best price/sensitivity compromise?

SE: I would take all three of them, 400, 500 and $600 \mathrm{MHz}$ are excellent instruments. They worked really nicely in the last years. It's really fun to work with them. My personal opinion is the stability and ease of use is higher at low fields while high field devices are more tedious to shim and the electronics is less stable. High field devices are more expensive. Sensitivity wise cooled probes and stronger magnets are better. But currently we are quantifying small organic molecules at $1 \mathrm{ppm}$ within 2 hours of measurement time at a $400 \mathrm{MHz}$ device. And this is very satisfying for our needs.

MS: What we have to think about here is that the instrument must be fit for purpose. If a $400 \mathrm{MHz}$ is fit for purpose for certain questions, then it's a $400 \mathrm{MHz}$. And in food, as I mentioned before, if we come with a $600 \mathrm{MHz}$ with one million dollars or whatever, then mostly the customer will turn away and say, "No, sorry. No way." So we have to live in this case with the $400 \mathrm{MHz}$. But we know the limitations, we know it can be done better. So if you have a requirement like in body fluids for example, like urine or so, I would immediately say, "Forget about $400 \mathrm{MHz}$, use $600 \mathrm{MHz}$ ", because the complexity is even higher.

But here really we have to ask the question, "What do we have to achieve?" And what is, let's say also, the boundary conditions? Sorry if I talk about cost here, but this is a very important aspect in this whole business of analytical services, for example. We must make sure we have added value compared to other cheaper methods, and this might justify the higher price. It might even justify a $600 \mathrm{MHz}$ eventually. But we have another thing that we are doing and this is we are developing platforms. So for food we have a $400 \mathrm{MHz}$ platform which has a lot of tools around it. For $600 \mathrm{MHz}$, this is the body fluid platform which also has dedicated tools. Because if we have to do the precision in quantification at every field strength, I will never be able to do... So then other people have to do it. But if we deliver a solution, then for food, this is if it's not a research system but a routine system which is used in regular screening, then it is a $400 \mathrm{MHz}$ and other people have the same instrument they can compare data. The idea of the platform and the cost.

Question from the audience: Concerning instrumentation, we experience quite some instability when we refill helium. How would you recommend to do deal with this regarding calibration? Because I really see this problem, but also when we change the probe head, we have for two or three days not the same machine. What would you do regarding these problems?

MS: Well, I think first of all the magnet is a living system and it's an individual, so not all magnets behave in the same way, so you have magnets you can fill helium. You could even measure while you're filling helium, they're so stable. Others are quite sensitive in this, and you have to accommodate that. So that means depending on what you want to do, you might have to wait for a certain time. Of course one could look if there is a reason for this, see if one can get rid of that, because normally if you change a probe it's not normal if you take that long time.

One would have to see if there is, I don't know, some moving parts there possible, or whatever it is, and vibrations, because helium gas builds up pressure, or nitrogen builds up pressure which is coming back in such problems. I think you should have somebody from us coming and look to what the reason is, instead of spending a lot of time waiting. I think we should find the reason for it. As I said, there might be a magnet which is very sensitive, but typically that's not. So even if you filled helium after three hours you should be able to continue.

Question from the audience: One further question concerning helium. I believe that the helium price will go up. Can Bruker please enlighten us if it's sensible to think about and to plan for a helium recovery system, when we have only one or two instruments?

MS: This really depends on the helium price. I think in the moment it is not justified to do so. And you have to also be aware that there is a pumping system which is introducing instabilities. We have improved a lot on that, but it still can be improved. So if you're not in a very remote location for example where helium is a problem, then I would say yes you need it, but otherwise I think in the moment and also for the foreseeable future, I would not expect the factor of four or five the helium to rise in price, and this is what you would have to calculate roughly. 


\section{Validation of qNMR}

DL: We now move to the validation and quality assurance of quantitative NMR. And, a nice question that was submitted was: "What is the best approach to determine the limit of detection". In light of our validation guidelines we have published ${ }^{25,26}$ I want to ask you, Torsten, perhaps you can enlighten us, what is the best way to do this? Is there a best way?

TS: I don't know if there's a best way. There are certain ways to do that. And whoever asked this question, please have a look on the validation guideline which we have written and is published in the Eurolab webpage ${ }^{25}$. It's a Eurolab technical report and so it's publicly accessible for free. And so there are certain ways to do that. One easiest thing is to do it visually, to say, "Okay, for this concentration I can see all my signals very clearly. I can distinguish this signal is triplet and I can see it clearly, and I can say, 'Okay, this concentration is my detection limit." You can do this also by this $\mathrm{S} / \mathrm{N}$ approach which is also used for chromatography to determine, or to say it should have, at least for the smaller signal, a S/N ratio of 3. Or you can do this by, I think it's a kind of regression by the DIN $32645^{27}$.

DL: The DIN $32645^{27}$ basically demands that you do an extra calibration curve in the range of the detection limits, and calculate the limits from the deviation of the fitted curve line. This is a German standard method.

TS: But all these possibilities are described in the Eurolab guideline ${ }^{25,26}$. The most critical parameter is the limit of quantification (LOQ), because I'm always in favour of not using the $S / N$ ratio of 10 , which the chromatography guys normally do. Because when you want to have an uncertainty level of $1 \%$ or so, your uncertainty would grow dramatically at this S/N level. The LOQ should depend somehow on your method's uncertainty. So it should not be defined by only this number of 10 for the $\mathrm{S} / \mathrm{N}$ ratio.

SE: The $\mathrm{ICH}$ guideline ${ }^{28}$ proposes these limits. For $\mathrm{S} / \mathrm{N}$ values, in principle the idea is that a signal with $\mathrm{SN}>3$ is defined as visible and therefore detectable. HPLC was also mentioned. A lot of the rules are meant for HPLC, historically. Impurities were quantified by HPLC and the formulas were applied first for HPLC, and they give a rough estimate where the limit of detection (LOD) might be, depending on the linearity data that is feed in. In NMR spectroscopy it's a little bit different. It's again, the really nice fact that it's inherently quantitative. The integral of signals is in most cases proportional to the height of the signal. And the height of the signal is the measure for the S/N value. Now we can simply use linearity data to back predict until we reach the point where we will not be able to see the signal anymore $(\mathrm{SN}<3)$. For NMR spectroscopy there's really great accordance between the integral and the height of the signal. This is the reason why LOD and LOQ values can be predicted with much higher reliability compared to HPLC or GC even without measuring samples at the respective concentration.

MS: I need to say something for food, because it's not so simple there. The problem is that we have a lot of overlap. Just think about the sugar area and think about that you have to identify sugar alcohol in the sugar area. Then when we talk about limit of detection of the quantification, this is not the limit that I would have for a free signal, this is quite different. The problem is this is different for every compound you want to look at, and this is why we have such a big effort. First of all you need to spike for ISO $17025^{29}$. You have to have 10 spikings manually but we also have some electronic spiking possibilities. This is the only way, I think, in these really complex mixtures how you get this problem solved reliably. So it's not the $\mathrm{S} / \mathrm{N}$ that I have, for example, on HMF there, this is a free signal, no problem, but as soon as I go into this area of large overlap, this is where it becomes complex, and this is why we also, for example, run a two-dimensional experiment. We run a very fast J-resolved with every sample, not only the 1D but also J-resolved. And we use that first for identification, because there's much less overlap. And then second, of course, it is used to feed also the quantification, of the deconvolution, because you know exactly where the position of your signal is and what the multiplicity is and the line shape. So you can take that as an information in addition. And I think this is what we have learned in these mixtures whether it's now food or body fluids. This is the only way how you get reliable data.

DL: The next question regarding validation perhaps Dr. Schütz wants to answer is regarding the accuracy that is required and how can this accuracy be ensured over a long time or for your single sample.

BS: I think all results should be accurate and only the precision, finally should be the question, how big is the precision? I think even if NMR is the primary method for quantification, validation of the quantification is necessary. So for the wine profiling, for example, we spike more than 1,000 samples manually in the chemistry lab. On top of that, of course, you can do artificial spiking experiments because NMR is a quantitative method and we could do it mathematically. But finally I think that we really should do it with real spiking and participate in interlaboratory tests. And finally it really depends on your problem, how big your precision, or how low the precision should be.

MS: You can spoil the NMR accuracy easily by using low cost tubes, because sometimes you have in the active area, you have variations... Let's say the diameter is different, and the wall thickness is different, and if you need, for example in wine, the ethanol needs about 1\%, you can spoil that just by using the wrong tube, and this is something to be considered.

DL: You actually anticipated the next question about the ethanol quantification. Apparently some labs have problems to quantify ethanol with NMR in beer. I cannot completely understand the problem because ethanol has such a large peak and we were able to quantify the alcoholic strength of beer ${ }^{3}$ already since 2004.

SE: I think there is one more point to consider regarding the last question related to the accuracy of ethanol. To put it simple, if something is not accurate, it's wrong, and being wrong is not okay. It's necessary to clarify whether we are talking about an internal standard method or an external standard method. I think so far the question of accuracy was discussed in the background of an external standard. First we measure the standard, then the beverage. And there are a lot of things that could be different between these two experiments and thereby influence the quantitation. In contrast, using the internal standard method only a single measurement is required. This reduces the possible sources of error drastically and would help to figure it out the source of error.

DL: I want to ask some further point on the accuracy topic to Torsten Schönberger. In food control we are normally pleased if the coefficient of variation (CV) is in the range of $5 \%$ to $10 \%$, this is okay for us. But I know that you achieve much, much lower CVs of $1 \%$ and less and this is necessary for your analysis. So how do you do it to achieve such low coefficients of variation?

TS: For the 1\%, it's nearly no problem. It just depends on the sample, I would say, because our powders what we have, there you can 
see, maybe five different ingredients, and this is not comparable to fruit juice, it's something completely different. So we can do really the targeted analysis, and we normally see separated signals which we can integrate or we can do some other tricks also. The other thing, where we want to do the high-precision measurement, there you have to be, of course, much more accurate. It begins with the weighing. You have to use an ultra-micro balance, and you have to be very careful, and you have to be very patient. You have to have a good day for doing the weighing. When you are a little bit hectic, you cannot do this. And you have to do all the steps, all the processing steps manually. Of course, the automation works quite well but not perfect. Some wrong phasing often can be observed or maybe some baseline problems and so on. You have to do it very carefully. You have to use a really broad integral region going over the satellites. But this is only possible when you have nearly pure compounds with only small impurities, but not for mixtures of common street samples also. And of course, one important thing, or the most important thing, when we talk about quantification is the $S / N$. When you want to achieve uncertainty level of $0.1 \%$, you need S/N of about 20,000. And if you are satisfied with $1 \%$, then you can use $\mathrm{S} / \mathrm{N}$ of 150 also. So it's really strongly depending on the S/N level, what uncertainty level you get afterwards.

Question from the audience: It's a comment, not a question, and a follow up on this accuracy question. So accuracy, it's all about error propagation. It's all the errors that we carry on during complete analysis process. I believe NMR is very precise but the accuracy really depends on who's using the instrument.

DL: And sample preparation, of course, probably has often higher errors than the actual NMR measurement.

Question from the audience: I have a couple of questions about the effect of the impact of the peak S/N ratio, on position. So, what is true of course that, smaller signals are less precise than highly intensive signal, but when you do this kind of correlation within the $\mathrm{S} / \mathrm{N}$, the peak of $\mathrm{S} / \mathrm{N}$ ratio and the error, is this validated by 10 repetitions, or is it just used automatically, based on experience, a large number of datasets? That's my first question. Second question is that, the nature of the noise, it's affected by the machines, and instruments, the operator, so a big thing... Even the definition of peak $\mathrm{S} / \mathrm{N}$ ratio, I mean in this case it's slightly different. So if you really want $0.1 \%$ precision, perhaps the measure of the noise should be investigated at some point. Do you know if anybody actually looked into this?

DL: I think these are interesting question, perhaps for the Bruker guys about the comparability between instruments, how calibrations compare between instruments, and I think you have also conducted some inter-laboratory trials. I know from own experience that qNMR is quite stable and it is possible to measure our wines on our own instrument, and get the spectrum to Bruker and still get out the accurate quantification data.

BS: This is due to the robust NMR system which we use, and in addition to the SOPs which we developed. It really can be that the development of SOPs which are robust, can take several months even. This includes the sample preparation, and the measurement and then of course finally, the data analysis of the samples. For us it's absolutely vital that, given different NMR machines of the same type, that they produce the very same NMR spectrum, otherwise we would not be able to measure and combine such big reference databases, and to develop our methods, and to sell those methods, if this cannot be possible. And we really took part in several dozens of such ring trials and interlaboratory tests; this we are doing even with every customer of us. And maybe one more comment regarding precision. Typically, in fruit juice and wine it is really possible to achieve for the normal compounds, not at the very lowest concentration, an intraday reproducibility of less than $0.4 \%$. This is possible with a very good preparation and automation measurement analysis, but of course it depends on the concentration of the compounds. So probably at very low concentrations it will be higher of course, but this is really possible.

Question from the audience: I would like to come back one more time to the 2D methods. You mentioned that in this case for the fruit juice, you have signal overlap which is a problem but you're using it for getting information, additional information. Now, how would you assess or grade the usefulness of directly using 2D methods for quantification? How does it compare to deconvolution of $1 \mathrm{D}$ spectra?

BS: So far, our results are that the quantification of $1 \mathrm{D}$ is more precise than the quantification of 2D. The quantification of 2D JRES is possible, and you also get accurate results. Of course we have to calibrate it, but you can calibrate it together with the 1D, so you don't have to spike something. I feel precision will be not so good as in the 1D, this are currently our results, and this is why we are currently only using the $2 \mathrm{D}$ for identification. Then we transfer this identification information to the 1D, then we perform the line fitting, but I would not say that this will be forever like this. I think there will be some substances which are easier to quantify in the $2 \mathrm{D}$, but so far we quantify in the 1D.

DL: I am absolutely agreeing, we are actually doing this 2D quantification in certain special cases, like for example, thujone in absinthe ${ }^{30}$, or taurine in energy drinks ${ }^{31}$, but we are now switching back to $1 \mathrm{D}$ with the line fitting, which I fully agree gives more accurate, precise results than the 2D quantification, but it can be done. It's doable, even with Topspin you can integrate the 2D peak. It's basically no problem, and it's the same procedure as for the 1D quantification.

MS: Let me just give you one example. If you talk about citrate in juices, depending on the ionic concentration, and the type of ions in there, this can be very broad, it can be sharp, and of course if it's broad the J-resolved will reduce it drastically. So you have to be very careful, if you do that, that you make sure that your lines are not dependent on the matrix for example. Then you will get a better result, but if it is dependent, it's not good. Question from the audience: My question is about standards, because we talked about internal and external standards. It's obvious that internal standard is the best, but if you cannot find the compound that is fit, where I can see, for this, or for example in some cases like, body fluids, some standards are not usable. So what is your solution, maybe some rating system?

BS: In fact, also in the food matrices, I think the TSP is not a good internal standard for quantification. In the fruit juice it really complexes, so in orange juice it is not usable at all, and even for apple juice, depending on the type or kind of apple juice, more than $20 \%$ of TSP complexes. This is better in wine, but even there the accuracy is worse compared to using an external standard sample. And this is what we are doing, and then we use the PULCON method, so we are optimising the $90^{\circ}$ pulse and according to that, the calibration of the quantification sample can be transferred to the actual sample, of course it is really important that you measure both samples with the very same NMR experiment, but to our 
knowledge the external calibration is the best way to achieve the best results.

Question from the audience: Considering the two types of applications of pharma and food, what are your recommendations? Do you recommend using standard integration, deconvolution, line fitting, what are your suggestions?

TS: If possible and you don't have any impurities in your signal and you have a pure signal, then you use, of course integration, normal integration, but if you have more overlap and you are forced to use the line fitting algorithm, which is normally less precise than integration but depends less on $\mathrm{S} / \mathrm{N}$ nearly not at all. So if your $\mathrm{S} / \mathrm{N}$ is less than 100 then you can also use the line fitting algorithm. We have also published one combination, one hybrid approach of integration and line fitting algorithm ${ }^{8}$, which is the best I would say if you want to be really, really precise and you want to calculate out the impurity signals in there. But okay, the simplest way of course is the integration, but sometimes you are forced to do some other evaluation and when your matrix is more complex, you have to use some kind of chemometric evaluation.

Question from the audience: What about this S/N? I mean the crudest point, the fact that the same molecule could have more than one signal, in order to get a different calculation for S/N. And the second question is, what about sequences such as Carr-Purcell-Meiboom-Gill (CPMG), in order to quantify metabolites in serum or plasma, because we are running a lot of metabolomics, and to detect the biomarkers in the blood. And usually, we are proposing some SOPs for doing NMR spectroscopy on blood and serum to do that, and there is a debate on classification and manipulation, and doing some kind of filtering of the growth signals by means of some techniques. What do you suggest as a SOP in this sphere?

MS: Of course the CPMG is also on option we could use for quantification. But you have to be aware that you need correction factors. This has to be done with spiking experiments that will make sure you hit the correct correction factors, therefore I think this is the way to go forward. You always have also to think with every additional step you do, you introduce variances, and also you introduce cost... So I'm coming back to my favourite subject.

But this is what we are dealing with everyday. So whenever it is possible to do it without any interaction like a filtering process, I would try to do it. But we also, for example revert to things like an SPE, extraction first, and bringing out a product very soon on this. Where we can also show that the reproducibility is not suffering, so that we are not compromising what we get from the NMR. In that case it's okay, and if it's justified, because we cannot get a good result otherwise, it is justified then we do it. But always ask yourself first, "Can it be done directly?"

In a CPMG I think it would be in the same position again that we are not talking about the pure $\mathrm{S} / \mathrm{N}$. Because the signal is still in an overlap situation and perhaps only broad lines have been removed. Question from the audience: You told that it is not always a generalised one, the $\mathrm{S} / \mathrm{N}$, because it depends on the overlaps and so on. So could you exploit the possibility to have available more than one signal, to develop this.

BS: For quantification of mixtures, you would have to apply line fitting algorithms, for at least $90 \%$ or $95 \%$ of the compounds. And those line fitting algorithms can be very complex. It is also possible that our methods do not only use one signal, but we do line fitting of several signals of the same compound, knowing all the interactions between the signals. Then finally, you have to validate this method, and you don't have to have a look to any $\mathrm{S} / \mathrm{N}$, but you have a look to your validation results of this more complex quantification method, and out of those validation results you can extract some limit of quantification. It is also needed to extract this limit of quantification by measuring hundreds of mixture samples because you have to know the variability of the overlapping signals in this region, and from that you have to measure several samples and extract the combined overall limit of quantification for your method.

\section{Conclusions and future perspectives}

DL: Thank you all. I think, this was our last question, but I do not want to finish without perhaps some remarks regarding the future. Perhaps Dr. Spraul, you can enlighten us what are the coming innovations, perhaps if you look 10 years ahead, what will qNMR be then?

MS: Of course there are different things we can work on, the first one is on the hardware side of things, so we can try to increase the $\mathrm{S} / \mathrm{N}$, which sometimes goes to where, let's say, stability as you mentioned in comparing medium to high field. So always the question is, "What compromise do we have to go?" Surely you can try very high $\mathrm{S} / \mathrm{N}$, and you can have cryo-probes. You have a very small diameter and they are extremely sensitive, but unfortunately for mixtures it doesn't help us, because we have only a small amount of sample. Because if you have an active volume of $350 \mu \mathrm{L}$ in a $5 \mathrm{~mm}$ tube, and you go down to $1.7 \mathrm{~mm}$ tube, it just has about $10-12 \mu \mathrm{L}$ active volume, then you already know what your result will be. So it makes no sense at all to use the small one, because you throw away S/N. I think we have to really work on, what is the need of the application? There surely is need for $1.7 \mathrm{~mm}$ cryo probe if you want to identify compounds, for example, which we have found in statistics, also in the food area to really differentiating between different situations, or country of origin, or type of variety, then of course we need to identify these things, and once we have identified them we can also quantify them. So I think one of the big tasks really in the future will be an even more of body fluids. There are so many unknown signals, and this is true for NMR and even more for MS, and here I actually have to vote also for combined usage of instrumentation. And we think that, for example, Fourier transform mass spectrometry will play a very important role in this area in the future, not necessarily for screening, but for actually identifying compounds that we don't know what they are. And there I see a challenge that we want to solve and we want to solve this integrated technology, NMR and mass spectrometry together. Of course for NMR and the screening I cannot do this, it's too expensive, also to have pure NMR systems, and if we can further evolve from the stability which already is extremely good but might become even better, I think we also have a very good chance to push that forward. It's just a few options on hardware side you can start to discuss about dynamic nuclear polarization, which for quantification probably is not the method of choice in a mixture if you want to do it on individual compounds only that might be a choice.

So there are things that might really revolutionize. And people are working on it, whether it comes to applicability and the problems we are currently discussing is a different question. And then we have the second side, and this is probably even more important, this is building databases of reference compounds, which are comparable to the spectra that you measure.

DL: This is also a large point on my wish list, such as, like in MS you could click on the signal and get a prediction what compound could 
this be. There are first developments in this direction, but this would really be extremely helpful.

MS: And then of course the analysis in terms of getting good quantification out of the mixture there, definitely we have to work further. We have solutions that are reliable but they need a lot of effort, and in terms of which parameters do I have to consider because the saturation effect if I'm close to the water. We have sometimes 50 different parameters which were influencing the quantification. To simplify that into a more robust and fast development methods, because we have to prove, unfortunately ISO 17025 they will ask you for that, and you have to give an answer. But there in the software development I think there is a huge question.

The other question for 2D would be, "Can we implement more of the fast sequences so that an HSQC for example becomes more viable?" In screening this is currently definitely not the case, it takes too long, unless I just want to look to major compounds in the mixture. So if we can do that, we can half the time, instead to double the number of scans. These are typical questions, and of course there are also the people that develop pulse sequences to be asked to contribute. So there are many possibilities how to improve.

DL: Perhaps I may ask the other experts the same question. What might happen in your field for this developmental stage of perhaps 10 years, what are your thoughts?

TS: Dirk, I don't want to refer to my field, but to a more general thing. I think Manfred Spraul already mentioned a lot of these technical things, and also in your introduction you mentioned that, I would say the modern qNMR method is maybe around 10 years old now. So the renaissance began in 2005, we heard about the reasons, and now we have these, but not really the acceptance in several areas; for example, we have the PANIC meeting in the United States, it's for practical applications, and there we have also a one-day validation workshop. And in this group we talked a lot about that exclusion, what the people say there. Because sometimes people go to court, and with the NMR value, the quantitative value, normally really reliable, but especially in the United States when people say, "Is it written somewhere in the SOPs or in the USP for the pharmaceuticals?" And we have to say, "No, it isn't." So it's not an accepted method and you cannot use this, and that's just the reason why we are just in the beginning of the development of qNMR. And I heard that also here from Germany about blood alcohol determination. There are guys who can do this with one drop of blood, but is not accepted, because they only want to use chromatography for this. And of course they want to earn money. And so we are working on some education campaign and so on and so on, but this will take more time. But what I actually want to say, I see a really good future for quantitative NMR and great developments, especially when I see all these applications here. From your lab (referring to DL) for example, or what I've seen here, these are really great applications and I think this will grow up much more in the future.

DL: Yes, I believe so, and I hope so as well. Perhaps Dr. Ehni wants to say something about the pharmaceutical industry to wrap up this session?

SE: In pharmaceutical industry the mixtures are not as complex as, for example, beer and wine. To facilitate quantitation, the existing qNMR experiments are satisfying already. But as mentioned by Torsten Schönberger, these methods need to find a way into the everyday analytics. A lobby for NMR as discussed at the PANIC meeting could help to familiarize authorities with NMR and increase the acceptance of the method. But this process might be a long and hard fight. qNMR is already widely used in the certification of assay reference standards. And also for identity standards. But for routine quantitation of small organic molecules there is still a large potential. I personally think this is worth it as qNMR is much faster and less error-prone when compared to HPLC or GC which require reference materials and method development for each single molecule to be quantified. This is the reason why qNMR can speed up the process of synthesis development enormously.

DL: Then last but not least, Dr. Schütz, from your side, what are the next food screeners we might expect seeing from Bruker?

BS: We have many plans for developing new methods, for example for edible oil.

MS: One last comment. It has to be $400 \mathrm{MHz}$, because we have such a large data matrix and we have people, groups that are working with us which are sitting exactly on the same protocols. If that is lost, a huge amount of money goes off. That's what we have to keep in mind.

DL: Thank you all so much for coming and staying till this late time, but I think it was an exciting topic and we have learned much about quantitative NMR. I really hope that our visions become true, that qNMR achieves official status and accepted methods, so that we can go to the courts with our NMR results. So thank you again, the experts and the audience, and have a nice evening in Karlsruhe.

\section{Acknowledgements}

Dr. Thomas Kuballa and Dr. Gisela Guthausen are thanked for conceiving of the discussion session and their help in structuring the questions. The conference participants are thanked for submitting written questions and contributing questions and comments during the session. Andreas Scharinger is thanked for excellent technical assistance in audio-recording and photographing the session. Scribie.com is acknowledged for the transcript of the audio recording.

\section{References}

1. S.K. Bharti and R. Roy, "Quantitative $1 \mathrm{H}$ NMR spectroscopy", TrAC Trends Anal. Chem. 35, 5-26 (2012). doi: http://dx.doi.org/10.1016/j. trac.2012.02.007

2. C. Simmler, J.G. Napolitano, J.B. McAlpine, S.N. Chen and G.F. Pauli, "Universal quantitative NMR analysis of complex natural samples", Curr. Opin. Biotechnol. 25, 51-59 (2014). doi: http://dx.doi.org/10.1016/j. copbio.2013.08.004

3. D.W. Lachenmeier, W. Frank, E. Humpfer, H. Schäfer, S. Keller, M. Mörtter and M. Spraul, "Quality control of beer using high-resolution nuclear magnetic resonance spectroscopy and multivariate analysis", Eur. Food Res. Technol. 220, 215-221 (2005). doi: http://dx.doi.org/10.1007/ s00217-004-1070-7

4. D.W. Lachenmeier, E. Humpfer, F. Fang, B. Schütz, P. Dvortsak, C. Sproll and M. Spraul, "NMR-spectroscopy for nontargeted screening and simultaneous quantification of health-relevant compounds in foods: the example of melamine", J. Agric. Food Chem. 57, 7194-7199 (2009). doi: http://dx.doi.org/10.1021/jf902038j

5. T. Schoenberger, "Determination of standard sample purity using the high-precision 1H-NMR process", Anal. Bioanal. Chem. 403, 247-254 (2012). doi: http://dx.doi.org/10.1007/s00216-012-5777-1

6. P.A. Hays and T. Schoenberger, "Uncertainty measurement for automated macro program-processed quantitative proton NMR spectra", 
Anal. Bioanal. Chem. 406, 7397-7400 (2014). doi: http://dx.doi, org/10.1007/s00216-014-8205-x

7. T. Schoenberger, U. Simmross and C. Poppe, "Forensic examination of electrical tapes using high resolution magic angle spinning (1)H NMR spectroscopy", Anal. Bioanal. Chem. 408, 123-129 (2016). doi: http:// dx. doi.org/10.1007/s00216-015-9126-Z

8. T. Schoenberger, S. Menges, M.A. Bernstein, M. Perez, F. Seoane, S. Sykora and C. Cobas, "Improving the performance of high-precision qNMR measurements by a double integration procedure in practical cases", Anal. Chem. 88, 3836-3843 (2016). doi: http://dx.doi. org/10.1021/acs.analchem.5b04911

9. S. Ehni and B. Luy, "A systematic approach for optimizing the robustness of pulse sequence elements with respect to couplings, offsets, and B1-field inhomogeneities (COB)", Magn. Reson. Chem. 50 Suppl 1, S63-S72 (2012). doi: http://dx.doi.org/10.1002/mrc.3846

10. S. Ehni and B. Luy, "BEBE(tr) and BUBI: J-compensated concurrent shaped pulses for $1 \mathrm{H}-13 \mathrm{C}$ experiments", J. Magn. Reson. 232, 7-17 (2013). doi: http://dx.doi.org/10.1016/j.jmr.2013.04.007

11. S. Ehni and B. Luy, "Robust INEPT and refocused INEPT transfer with compensation of a wide range of couplings, offsets, and B1-field inhomogeneities (COB3)", J. Magn. Reson. 247, 111-117 (2014). doi: http:// dx.doi.org/10.1016/j.jmr.2014.07.010

12. M. Spraul, M. Hofmann, P. Dvortsak, J.K. Nicholson and I.D. Wilson, "Liquid chromatography coupled with high-field proton NMR for profiling human urine for endogenous compounds and drug metabolites", J. Pharm. Biomed. Anal. 10, 601-605 (1992). doi: http://dx.doi. org/10.1016/0731-7085(92)80086-3

13. M. Spraul, P. Neidig, U. Klauck, P. Kessler, E. Holmes, J.K. Nicholson, B.C. Sweatman, S.R. Salman, R.D. Farrant, E. Rahr, C.R. Beddell, and J.C. Lindon, "Automatic reduction of NMR spectroscopic data for statistical and pattern recognition classification of samples", J. Pharm. Biomed. Anal. 12, 1215-1225 (1994). doi: http://dx.doi. org/10.1016/0731-7085(94)00073-5

14. P.S. Belton, I. Delgadillo, E. Holmes, A. Nicholls, J.K. Nicholson and M. Spraul, "Use of high-field ${ }^{1} \mathrm{H}$ NMR spectroscopy for the analysis of liquid foods", J. Agric. Food Chem. 44, 1483-1487 (1996). doi: http://dx.doi. org/10.1021/jf950640z

15. P.S. Belton, I.J. Colquhoun, E.K. Kemsley, I. Delgadillo, P. Roma, M.J. Dennis, M. Sharman, E. Holmes, J.K. Nicholson and M. Spraul, "Application of chemometrics to the $\mathrm{H}-1$ NMR spectra of apple juices: discrimination between apple varieties", Food Chem. 61, 207-213 (1998). doi: http://dx.doi.org/10.1016/S0308-8146(97)00103-9

16. P. Rinke and M. Spraul, "NMR Analysis. Successful application of SGFProfiling", New Food 11, 18-23 (2008).

17. M. Spraul, E. Humpfer, H. Schäfer, B. Schütz, M. Mörtter and P. Rinke, "NMR-based mixture analysis on the example of fruit juice quality control using statistics and quantification" in NMR spectroscopy in pharmaceutical analysis, Ed by U. Holzgrabe, I. Wawer and B. Diehl. Elsevier, Amsterdam, the Netherlands, p. 319-339 (2008). doi: http://dx.doi. org/10.1016/B978-0-444-53173-5.00013-5

18. M. Spraul, B. Schütz, E. Humpfer, M. Mörtter, H. Schäfer, S. Koswig and P. Rinke, "Mixture analysis by NMR as applied to fruit juice quality control", Magn. Reson. Chem. 47 Suppl 1, S130-S137 (2009). doi: http:// dx.doi.org/10.1002/mrc.2528

19. R. Godelmann, F. Fang, E. Humpfer, B. Schütz, M. Bansbach, H. Schäfer and M. Spraul, "Targeted and nontargeted wine analysis by ${ }^{1} \mathrm{H}$ NMR spectroscopy combined with multivariate statistical analysis.
Differentiation of important parameters: grape variety, geographical origin, year of vintage", J. Agric. Food Chem. 61, 5610-5619 (2013). doi: http://dx.doi.org/10.1021/jf400800d

20. J.K. Nicholson, P.J. Foxall, M. Spraul, R.D. Farrant and J.C. Lindon, "750 MHz ${ }^{1} \mathrm{H}$ and ${ }^{1} \mathrm{H}-{ }^{13} \mathrm{C}$ NMR spectroscopy of human blood plasma", Anal. Chem. 67, 793-811 (1995). doi: http://dx.doi.org/10.1021/ ac00101a004

21. Y.B. Monakhova, B. Schütz, H. Schäfer, M. Spraul, T. Kuballa, H. Hahn and D.W. Lachenmeier, "Validation studies for multicomponent quantitative NMR analysis: the example of apple fruit juice", Accredit. Qual. Assur. 19, 17-29 (2014). doi: http://dx.doi.org/10.1007/s00769-0131026-3

22. P. Maes, Y.B. Monakhova, T. Kuballa, H. Reusch and D.W. Lachenmeier, "Qualitative and quantitative control of carbonated cola beverages using ${ }^{1}$ H NMR spectroscopy”, J. Agric. Food Chem. 60, 2778-2784 (2012). doi: http://dx.doi.org/10.1021/jf204777m

23. Y.B. Monakhova, H. Schäfer, E. Humpfer, M. Spraul, T. Kuballa and D.W. Lachenmeier, "Application of automated eightfold suppression of water and ethanol signals in ${ }^{1} \mathrm{H}$ NMR to provide sensitivity for analyzing alcoholic beverages", Magn. Reson. Chem. 49, 734-739 (2011). doi: http://dx.doi.org/10.1002/mrc.2823

24. Y.B. Monakhova, S.P. Mushtakova, T. Kuballa and D.W. Lachenmeier, "Investigation into the structural composition of hydroalcoholic solutions as basis for the development of multiple suppression pulse sequences for NMR measurement of alcoholic beverages", Magn. Reson. Chem. 52, 755-759 (2014). doi: http://dx.doi.org/10.1002/mrc.4129

25. T. Schönberger, Y.B. Monakhova, D.W. Lachenmeier, T. Kuballa, NonProfit Expert Team (NEXT)-NMR working group Germany, Guide to NMR Method Development and Validation - Part I: Identification and Quantification. Eurolab Technical Report No. 01/2014. EUROLAB General Secretariat, 2014. doi: http://dx.doi.org/10.13140/RG.2.1.1244.3689

26. T. Schönberger, Y.B. Monakhova, D.W. Lachenmeier, S. Walch, T. Kuballa, Non-Profit Expert Team (NEXT)-NMR working group Germany, Guide to NMR Method Development and Validation - Part II: Multivariate data analysis. Eurolab Technical Report No. 01/2015. EUROLAB General Secretariat, 2015. doi: http://dx.doi.org/10.13140/RG.2.1.4265.1289

27. Deutsches Institut für Normung, DIN 32645. Chemical analysis Decision limit, detection limit and determination limit under repeatability conditions - Terms, methods, evaluation. Beuth Verlag, Berlin, Germany, 2008.

28. ICH, International conference on harmonisation of technical requirements for registration of pharmaceuticals for human use. ICH harmonised tripartite guideline. Validation of analytical procedures: text and methodology Q2(R1). International Council for Harmonisation (ICH), Geneva, Switzerland, 2005.

29. ISO, ISO/IEC 17025:2005 General requirements for the competence of testing and calibration laboratories. International Organization for Standardization, Geneva, Switzerland, 2005.

30. Y.B. Monakhova, T. Kuballa and D.W. Lachenmeier, "Rapid determination of total thujone in absinthe using $1 \mathrm{H}$ NMR spectroscopy", Int. J. Spectrosc. 2011, Article ID 171684 (2011). doi: http://dx.doi. org/10.1155/2011/171684

31. K. Wegert, Y.B. Monakhova, T. Kuballa, H. Reusch, G. Winkler and D.W. Lachenmeier, "Regulatory control of energy drinks using $1 \mathrm{H}$ NMR spectroscopy”, Lebensmittelchem. 66, 143-145 (2012). doi: http://dx.doi. org/10.1002/lemi.201290113 